Planetary Systems in the Universe - Observation, Formation and Evolution

Proceedings IAU Symposium No. 202, (c)2004 IAU

Alan Penny, Pawel Artymowicz, Anne-Marie Lagrange, 8 Sara Russell, eds.

\title{
A Comparative Anatomy of Dusty Disks Imaged by NICMOS $^{1}$
}

\author{
Glenn Schneider, Dean C. Hines, Murray Silverstone \\ Steward Obs., U. Az., 933 N. Cherry Ave., Tucson, AZ, 85721 USA
}

\author{
Alycia J. Weinberger \\ Dept. of Physics 8 Astronomy, UCLA, Los Angeles, CA, 90095 USA
}

\author{
and the NICMOS/IDT EONS Team
}

\begin{abstract}
Using the Near Infrared Camera and Multi-Object Spectrometer (NICMOS) on the Hubble Space Telescope we have conducted a coronagraphic imaging survey of 18 main sequence stars with large infrared excesses, searching for circumstellar dust (debris) in scattered light. Dusty disks with radial and hemispheric brightness anisotropies and complex morphologies, both possibly indicative of dynamical interactions with unseen planetary mass companions, were spatially resolved and imaged around three young ( $\$ 10 \mathrm{Myr}$ old) stars. From these observations we describe the debris systems around: a) HR 4796A (A0V), a $70 \mathrm{AU}$ radius ring less than $14 \mathrm{AU}$ wide with unequal ansal flux densities; b) HD 141569A (Herbig Ae/Be), a 400 AU radius disk with a 40 AU wide gap; and c) TW Hya (K7 T-Tauri), a pole-on circularly symmetric disk with a radial break in its surface density of scattering particles. Additionally, our nondetection of scattered light and high precision photometry of a fourth system of similar age, HD $98800 \mathrm{~A} / \mathrm{B}$, coupled with mid and thermal IR measurements, greatly constrain a likely model for the debris about the B component.
\end{abstract}

\section{Introduction}

The successful imaging of the $\beta$ Pictoris debris disk by Smith \& Terille (1984) established the paradigm of the evolution of dusty circumstellar environments as a likely consequence of planet-building. Until recently, however, the ability to test the competing contemporary theories of disk evolution, planet formation, and disk/planet interactions has been observationally challenged. With the advent of spaced-based coronagraphy, the morphological diversity in debris disk structures, which theory must explain, has become evident. Here we discuss the properties of dusty debris systems of similar ages $(\lesssim 10 \mathrm{Myr})$, observed with NICMOS, which span two orders of magnitude in physical size.

\footnotetext{
${ }^{1}$ PowerPoint ${ }^{\mathrm{TM}}$ presentation: http://nicmosis.as.arizona.edu:8000/TALKS/IAU202.PPT
} 


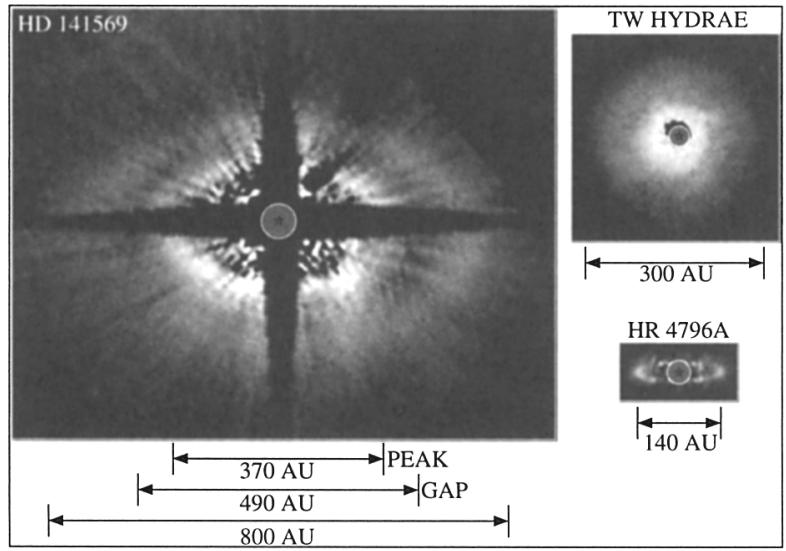

Figure 1. Debris disks imaged coronagraphically by NICMOS reveal a diversity in sizes, structures, complexities, and anisotropies.

\section{HR 4796A - A Very Dusty, Confined, Debris Ring}

HR $4796 \mathrm{~A}$ is an A0V star with an estimated age of $8 \pm 3$ Myr (Stauffer et al. 1995). At a distance of $67 \pm 3 \mathrm{pc}$, HR $4796 \mathrm{~A}$ is a member of the TW Hydrae association (Webb et al. 1999), the nearest site of recent star formation to the Earth. The presence of a large amount of circumstellar dust around HR 4796A was inferred from its IRAS infrared excess by Jura (1991) who estimated the optical depth due to dust, $\tau_{\text {dust }}=L_{\text {disk }} / L_{*} \sim 5 \times 10^{-3}$, to be approximately twice that of $\beta$ Pictoris. Jura et al. (1995) noted that his earlier estimate of the dust temperature of $110 \mathrm{~K}$ indicated a lack of material at radii $<40 \mathrm{AU}$ from the star, and that grain sizes of $z 3 \mu \mathrm{m}$ were required for the dust to be gravitationally bound at radii from 40-200 AU. Beuzit et al. (1997) attempted to image the disk, in the near-IR ( $\mathrm{K}^{\prime}$ bandpass) without success using a ground-based coronagraph with adaptive optics. Subsequently, Koerner et al. (1998) and Jayawardhana et al. (1998) independently imaged the disk in the mid-IR. An inner depleted region, as predicted by Jura, was evident in Koerner et al.'s high resolution $20.8 \mu \mathrm{m}$ observations, which was reproduced by a model suggesting a disk with: $\mathrm{i}=72_{-9}^{+6 \circ}, \mathrm{PA}=28^{\circ} \pm 6^{\circ}, R_{\text {inner }} \sim 55 \mathrm{AU}$, and $R_{\text {outer }} \sim 80 \mathrm{AU}$, which they suggested was a Kuiper belt-like dust ring.

Schneider et al. (1999) obtained well-resolved NICMOS coronagraphic images of the disk and reported on its morphology and photometry from $1.6 \mu \mathrm{m}$, and subsequent higher $\left(0{ }^{\prime \prime} 1\right)$ resolution $1.1 \mu \mathrm{m}$ observations. The near-IR light scattered by the HR 4796A circumstellar dust is narrowly confined in a ring $<0$ "' 21 (14AU) wide with a diameter:width ratio of $\sim 10: 1$, peaking in brightness at a radius of $1^{\prime \prime} .05$ (70AU) from the star. The dust ring presumably arises from the continuous collisional erosion of planetesimals because the time-scale for clearing of such particles due to P-R drag is a few Myr. The narrowness of the ring may implicate the dynamical confinement of the dust particles by one or more unseen (planetary mass) bodies, perhaps by "shepherding" (Goldrich \& Tremain 1979), though the physically associated B component of the system $7{ }^{\prime \prime} 7$ away might play a role in the outer truncation of the disk. The disk-scattered near-IR flux is in good agreement with absorption in the visible and re-radiation in the mid- 


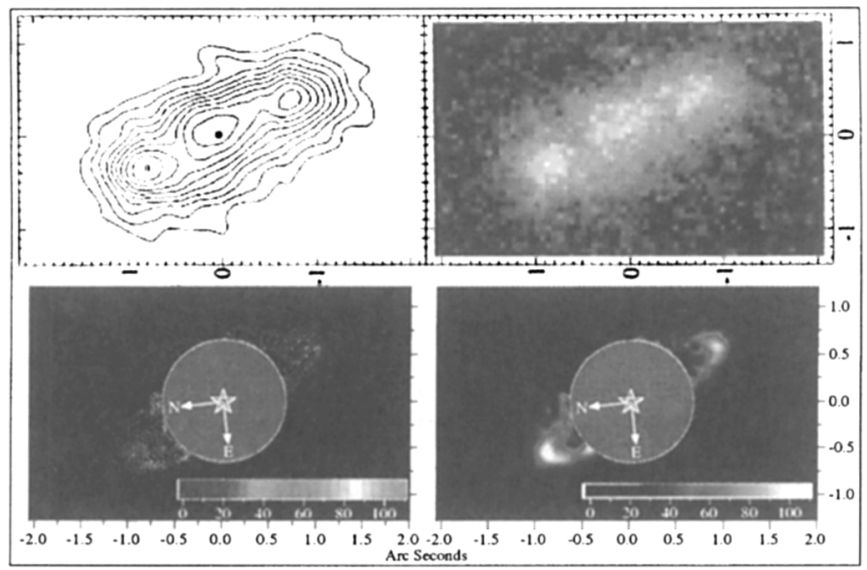

Figure 2. The HR 4796A debris disk as seen in thermal emission at $18.2 \mu \mathrm{m}$ (top - OSCIR image from Telesco et al. (2000)) and scattered light at $1.1 \mu \mathrm{m}$ (bottom - NICMOS image from Schneider et al. (1999).

IR, with flux densities indicating optical depths due to dust of $\sim 1.4 \pm 0.2 \times 10^{-3}$ at $1.1 \mu \mathrm{m}$, and $2.4 \pm 0.5 \times 10^{-3}$ at $1.6 \mu \mathrm{m}$. The somewhat red color of the grains is likely indicative of a mean particle size of at least a few microns and not trapped interstellar dust.

An asymmetry in the brightness of the $\mathrm{NE}$ and SW ansae of the ring of $\sim 10-15 \%$ was noted in the NICMOS images at both 1.1 and $1.6 \mu \mathrm{m}$. A similar asymmetry was seen by Telesco et al. (2000) in deep 10.8 and $18.2 \mu \mathrm{m}$ images. Further, they confirmed that the central hole is largely cleared. The inward radial fall-off from the $70 \mathrm{AU}$ radius ring is shallower at $18.2 \mu \mathrm{m}$ then inferred from the NICMOS images, reaching a depth of $\lesssim 3 \%$ of the ansal flux in the central zone. Telesco et al. (2000) speculate that the E/W brightness anisotropies might implicate the existence of a gravitational perturber causing the observed "pericenter glow" (Wyatt et al. 2000) from particles in the NE side of the disk shifted closer to the star.

With the geometry of the scattered-light disk established by Schneider et al. (1999), Augereau et al. (1999) re-reduced the Beuzit et al. (1997) K' observations and detected the ring ansae. They then developed a two-component model to reproduce all the then-available observations, from near-IR to millimeter wavelengths. To fit both the brightness distribution of the cold dust and the SED of the hot dust they employed a) cold amorphous ( $\mathrm{Si}$ and $\mathrm{H}_{2} \mathrm{O}$ ice) grains $>10 \mu \mathrm{m}$ in size with porosity $\sim 0.6$, peaking at 70AU, and b) hot dust at $\sim 9 \mathrm{AU}$ of "comet-like" composition (crystalline $\mathrm{Si}$ and $\mathrm{H}_{2} \mathrm{O}$ ), with porosity of $\sim 0.97$. Their model gives rise to a minimum dust mass of $\sim 4$ Earth masses with a gas:dust ratio $<1$.

Greaves et al. (2000) obtained JCMT/SCUBA 450 and $850 \mu \mathrm{m}$ flux excess measures of 0.18 and $0.019 \mathrm{Jy}$, respectively. They estimated the total gas mass in the disk to be < 1-7 Earth masses, consistent with Augereau et al. (1999).

Kenyon et al. (1999) evolved a model disk using a "particle in a box" coagulation code to fit the observed $1.1 \mu \mathrm{m}$ brightness distribution. In doing so they assumed isotropic scattering with albedo of 0.3 (Augereau et al. 1999) 
and adjusted $\tau$ to obtain a near-IR optical depth of $1.5 \times 10^{-3}$. They found that planet formation at 70AU in $10 \mathrm{Myr}$ is possible assuming an initial disk of a 10 20 minimum-mass solar nebula (MMSN), and that dust production associated with planet formation is then confined to a ring with $\Delta=7-15$ AU. The optical depth in the ring satisfies constraints on scattered light at $1-2 \mu \mathrm{m}$ and on the thermal emission at 10-100 $\mu \mathrm{m}$ if the dust size distribution is $\mathrm{N} \sim r_{\mathrm{i}}{ }^{-q}$ with $\mathrm{q} z 3$ for $r_{\mathrm{i}}=1 \mathrm{~m}$. Further, they note that initial disk masses smaller than $10_{M M S N}$ fail to produce planets and an observable dusty ring in $10 \mathrm{Myr}$.

\section{HD 141569A - A Younger ( 5Myr) Dusty Disk}

Mid-IR observations of the Herbig Ae/Be star and IRAS excess source HD 141569A (B9Ve, d 100pc) by Silverstone et al. (1998) and Fisher et al. (2000) showed an elliptical hot dust component extending to $\sim 1$ " along the disk major axis. NICMOS $1.1 \mu \mathrm{m}$ coronagraphic observations revealed a scattered light component to the circumstellar disk outside of the region of mid-IR emission to a radius of at least $400 \mathrm{AU}$. The disk exhibits a complex morphology including a $40 \mathrm{AU}$ wide gap in the surface brightness profile at a radius of 250AU (Weinberger et al. 1999a) and an outer peak at 340AU. The scattered light disk was independently detected with lower resolution (0"17) NICMOS coronagraphic observations by Augereau et al (1999), which confirmed the global structure of the outer portions of the disk.

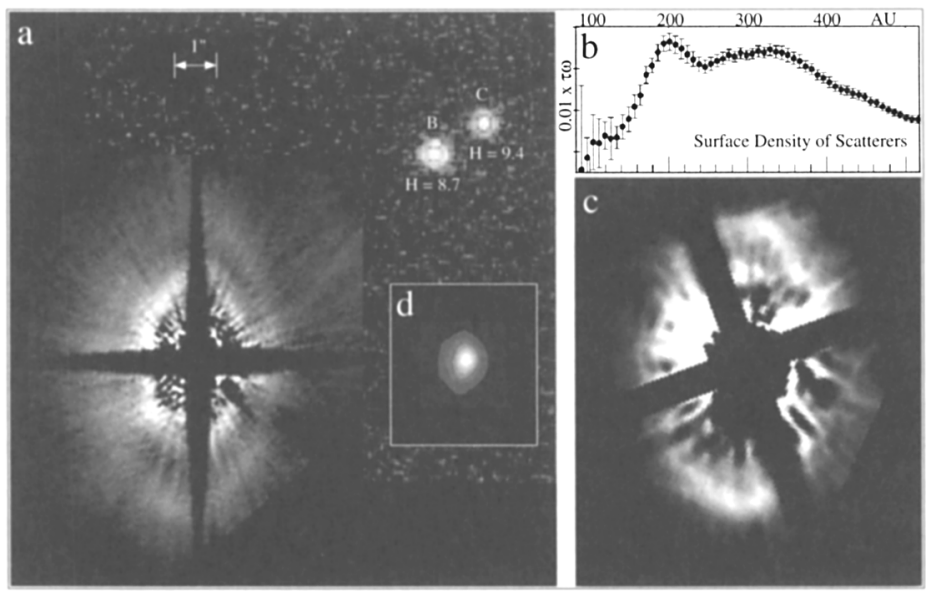

Figure 3. HD 141569. a) NICMOS $1.1 \mu \mathrm{m}$ coronagraphic image of the disk astrometrically registered with $1.7 \mu \mathrm{m}$ acquisition images of the $\mathrm{B} / \mathrm{C}$ companions. b) Radial dependence of the surface density of $1.1 \mu \mathrm{m}$ scatterers (Weinberger et al. (1999a). c) NICMOS $1.6 \mu \mathrm{m}$ coronagraphic image (Augereau et al. (1999a), d) Keck/MERLIN $12.5 \mu \mathrm{m}$ image showing thermal-IR emission interior to light scattering by cold dust (Silverstone et al. 1998). All images (and profile) are PSF-subtracted and are shown at the same scale with North up.

The disk, with a $1.1 \mu \mathrm{m}$ total flux density of $8 \pm 2 \mathrm{mJy}$ beyond 0.6 (inner peak surface brightness $0.3 \mathrm{mJy} \operatorname{arcsec}^{-2}$ at $185 \mathrm{AU}$ ) is inclined to our line-of- 
sight by $51^{\circ} \pm 3^{\circ}$. From the mid and near-IR flux densities the global albedo is estimated as $\gtrsim 0.35$. The intrinsic scattering function of the dust results in a brightness anisotropy in the ratio of $1.5 \pm 0.2$ with the brighter side in the direction of forward scattering. The region of the gap, at a radius of $250 \mathrm{AU}$, may be partially cleared of material by an unseen co-orbital planetary companion. If so the width/radius ratio of the gap implies a planetary mass of $\sim 1.3$ Jupiters. This is consistent with our 2 Jupiter mass detection limit at this radius.

HD $141569 \mathrm{~A}$ is the brightest member of an $\gtrsim 6.5: 1$ hierarchical triple system $\left(\Delta \mathrm{A}(\mathrm{BC})=8 \prime \prime 3, \Delta \mathrm{BC}=1^{\prime \prime} \cdot 3\right)$ as reported by Weinberger et al. (2000). The physical companions (confirmed by common proper motions and radial velocities) likely influence the dynamics of the circumstellar disk. The surface density of scatterers is equal at 200 and $360 \mathrm{AU}$. If the barycenter of the companion orbit is not coplaner with the disk then significant vertical velocities could be excited in the disk particles. The circularity of the gap implies dynamical stability on long timescales, and continual clearing is required to remove Poynting-Robertson and radiation-pressure driven particles transiting the gap.

Recent ground-based spectroscopy of the companions reveal them to be young M dwarf stars (M2V and M4V), with estimated ages of $\sim 5 \pm 3 \mathrm{Myr}$ (Weinberger et al. 2000) based upon $\mathrm{H} \alpha$ emission and Lithium equivalent widths. Assuming coevality, these measures further constrain the age of the disk, which might be a factor of $\sim 2$ younger than the HR $4796 \mathrm{~A}$ disk, but still may show evidence of planetary dynamical interactions at this earlier "evolutionary" epoch.

\section{TW Hydrae - An Optically Thick Disk}

TW Hydrae is an isolated classical T-Tauri star (K7Ve; Rucinski \& Krauter 1983 ) at a distance of $56 \pm 7 \mathrm{pc}$ (as measured by Hipparcos) and a member of the young stellar association which bears its name. With an estimated age of $\sim 8 \mathrm{Myr}$, TW Hya exhibits $\mathrm{H} \alpha$ and UV excesses, and is X-ray bright, as is typical for T-Tauri stars. TW Hya was identified by IRAS as an IR excess star with $L_{\text {disk }} / L_{*} \sim 0.3$. Both sub-millimeter continuum and $\mathrm{CO}$ emission have been observed (Weintraub et al. 1989). The presence of a disk with a deficient gaseous component (compared to primordial disks) was established by Zuckerman et al. 1995 and Kastner et al. 1997. Recently, Weintraub, Kastner \& Barry (2000) detected emission from the 1-0 S(1) line of $\mathrm{H}_{2}$. The $\mathrm{H}_{2}$ emission is consistent with expectations of X-ray excitation and is an important tracer for possibly establishing the gas-to-dust ratios in late T-Tauri disks.

TW Hya was found to harbor a bright, face-on, optically-thick disk seen in NICMOS 1.1 and $1.6 \mu \mathrm{m}$ coronagraphic images to a radius of $\sim 190 \mathrm{AU}$ (Weinberger et al. 1999b). Krist et al. (2000) independently imaged the disk at 0.6 and $0.8 \mu \mathrm{m}$ with WFPC-2. The face-on geometry of the optically-thick disk, seen to large radii, implies that the disk must be outwardly flared. An $\mathrm{r}^{-2.6}$ dependence in the surface brightness is seen from $\sim 45-130 \mathrm{AU}$ in both near-IR colors, but departs from a simple power-law fit for $\mathrm{r}>90 \mathrm{AU}$ at $1.6 \mu \mathrm{m}$. Similar deviations, which may be indicative of dynamical sculpting of the grains, are also seen in the optical. When corrected for the color of the star, the disk exhibits essentially gray scattering in the near-IR (with a color index uncertainty of $\sim 0.2$ ). 


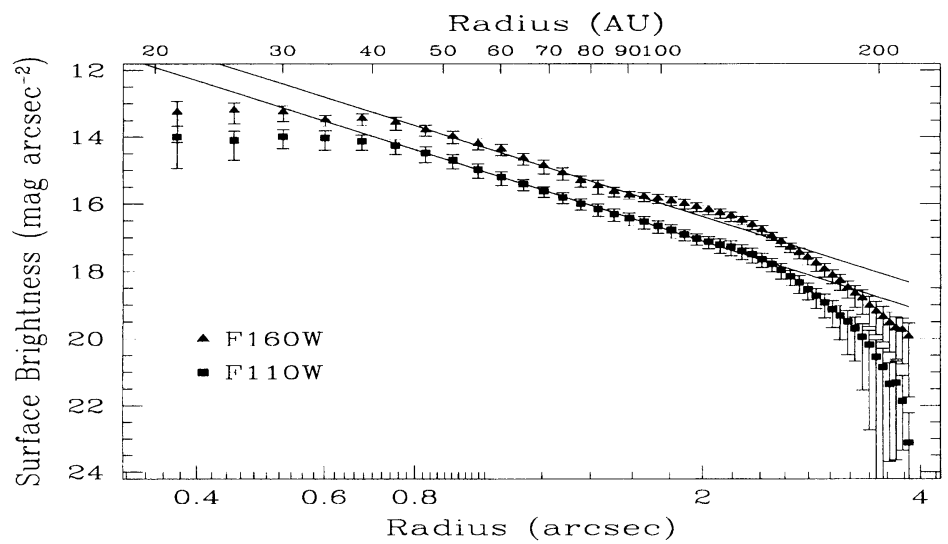

Figure 4. TW Hya surface brightness profile (Weinberger et al. 1999b).

\section{HD 98800 - A Remarkable Quadruple System}

HD $98800 \mathrm{~A} / \mathrm{B}$ is a hierarchical quadruple system composed of two spectroscopic binaries with "similar" primaries, both previously classified as pre-main sequence $\mathrm{K}$ stars. Also a member of the TW Hydra association, and hence approximately $8 \mathrm{Myr}$ of age, the HD 98800 system is at a distance of $47 \pm 6 \mathrm{pc}$. The primary components of the spectroscopic pairs are currently separated by $0 ! 8$, so they are easily resolved by HST at all accessible wavelengths. The low-mass spectroscopic companions orbit with periods of $(\mathrm{Aa}+\mathrm{Ab})=262$ days and $(\mathrm{Ba}+\mathrm{Bb})=315$ days (Torres et al. 1995) with separations of $\sim 1 \mathrm{AU}$ from their primaries.

IRAS found the HD 98800 system to contain one of the brightest planetary debris systems (PDS) in the sky (Zuckerman \& Becklin 1993). Gerhz et al. (1999) showed that the PDS is centered on the B component from 4.7 and $9.8 \mu \mathrm{m}$ observations. Twenty percent of the luminosity from B is emitted in a $164 \pm 5 \mathrm{~K}$ SED from mid-IR to sub-mm wavelengths. With high-precision near-IR photometry of the stars Low, Hines \& Schneider (1999) found $\mathrm{T}_{\text {eff }}(\mathrm{A})=3831 \pm 55 \mathrm{~K}$ and $\mathrm{T}_{e f f}(\mathrm{~B})=3459 \pm 37 \mathrm{~K}$, and the scattered:total light ratio from $\mathrm{B}<6 \%$, implying $\omega(\mathrm{PDS})<0.3$. They suggest a PDS with an "equivalent" radius $=2 \mathrm{AU}$, an actual $R_{\text {inner }} \sim 4.5 \mathrm{AU}$, subtending no more than $20 \%$ of the sky seen from the stars (i.e., an effective cross-section of $\lesssim 17 \mathrm{AU}^{2}$ ). No near-IR flux excess was detected in either high-resolution direct imaging as reported by Low et al. (1999), or coronagraphically at $1.6 \mu \mathrm{m}$ (masked to $0.3,14 \mathrm{AU}$ ), further suggesting the dust must be in the close environs of the B component. The PDS about the B component may be similar to the debris around our Sun as it might have appeared a few million years its after formation.

Koerner et al. (2000) confirmed the circumbinary disk about $(\mathrm{Ba}+\mathrm{Bb})$, and demonstrated that it accounts for all of the excess including a significant silicate feature. From mid-IR imaging they suggest a disk with $R_{\text {inner }}=5.0 \pm 2 \mathrm{AU}$, a total cross-section of the grains $=16 \pm 3 \mathrm{AU}^{2}$, and a disk mass of $0.001-0.1 \mathrm{M}_{\mathrm{MOON}}$.

Fully deblended and well-resolved STIS spectra recently obtained by Schneider et al. (2000) are consistent with the photometrically determined effective temperatures and derived luminosities, and indicate that the B (dust enshrouded) component is likely an M0V star rather than late K. Existing PMS 


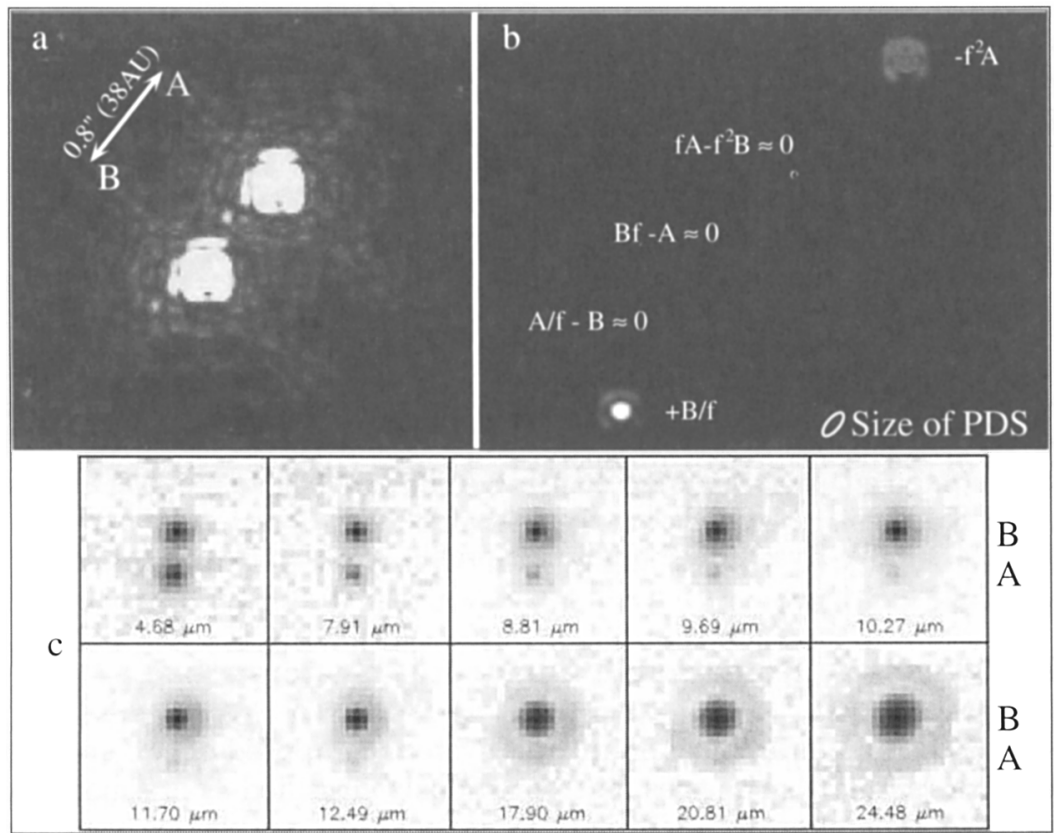

Figure 5. No scattered light excess was detected in (a) NICMOS highresolution $\left(\lambda / 2 \mathrm{D}=00^{\prime \prime} 041,2 \mathrm{AU}\right)$ images at $0.95 \mu \mathrm{m}$ by (b) replicate scaled $\mathrm{PSF}$-subtraction. Increasing thermal emission is seen from dust around the $\mathrm{B}$ component (c), as the photospheric flux from A declines, with increasing wavelength from $5-25 \mu \mathrm{m}$ (Koerner et al. 1999).

models give reasonable masses for these stars, but are inconsistent with age estimates derived from observations as noted by Low et al. (1999).

\section{Summary}

Each of the debris disks observed by NICMOS exhibit properties and characteristics which may be attributed to co-spatial planet-building. Along with $\beta$ Pic and its five-fold disk asymmetries (Kalas \& Jewitt 1995), the complex structures seen in the AB Aur and HD 163296 disks (Grady et al. 1999a,b), and the recent imaging of a debris disk around HD 100546 (Pantin, Waelkens \& Lagage (2000); Augereau et al. 2000), these observations begin to form a basis upon which the competing theories of disk/planet interactions and formation may be tested. This sample, however, is very small. Establishing the validity of proposed evolutionary models will require a significant increase in the number of spatially resolved images of disks, and more precise determinations of their ages.

This work is supported by NASA grant NAG 5-3042 to the NICMOS/IDT.

\section{References}

Augereau, J. C., et al. 1999a, A\&A, 350, L51 
Augereau, J. C., et al. 2000, A\&A, in press

Augereau, J. C., et al. 1999b, A\&A, 348, 557

Beuzit, J., et al. 1997, A\&AS, 125, 175

Fisher, R. S., et al. 2000, ApJ, 532, L141

Gehrz, R. D., et al. 1999, AAS Meeting, 195, 7812

Goldreich, P., \& Tremaine, S. 1979, Nature, 277, 97

Grady, C.A., et al. 1999a, ApJ, 523, L151

Grady, C.A., et al. 1999b, AAS Meeting, 195, 0208

Greaves, J. S., Mannings, V. \& Holland, W. S. 2000, Icarus, 143, 155

Jayawardhana, et al. 1998, ApJ, 503, L79

Jura, M. 1991, ApJ, 383, L79

Jura, M., et al. 1995, ApJ, 445, 451

Kastner, J. H., et al. 1997, Science, 277, 67

Kalas, P. \& Jewitt, D. 1995, AJ, 110, 794

Kenyon, et al. 1999, ApJ, 524, L119

Koerner, D. W., et al. 1998, ApJ, 503, L83

Koerner, D. W., et al. 2000, ApJ, 533, L37

Krist, J. E., et al. 2000, ApJ, 538, 793

Pantin, E., Waelkens, C. \& Lagage, P. O. 2000, A\&A, 361, L9

Low, F. J., Hines, D. C., \& Schneider, G. 1999, ApJ, 520, L45

Rucinski, S.M., \& Krautter, J. 1983, A\&A, 121, 217

Schneider, G. et al. 1999, ApJ, 513, L127

Schneider, G. et al. 2000, Lecture Notes in Physics, (Springer-Verlag), in press.

Silverstone, M. D. et al. 1998, AAS Meeting, 193, 7316

Smith, B. A., \& Terrile, R. J. 1984, Science, 226, 1421

Stauffer, J.R., Hartmann, L.W. \& Barrado y Navascues, D. 1995, ApJ, 454, 910

Telesco, C. M. et al. 2000, ApJ, 530, 329

Torres, G., et al. 1995, ApJ, 452, 870

Webb, R. A., et al. 1999, ApJ, 512, L63

Weinberger, A. J., et al. 1999a, ApJ, 525, L53

Weinberger, A. J., et al. 1999b, AAS Meeting, 194, 6904

Weinberger, A. J., et al. 2000, ApJ, in press

Weintraub, D. A., Sandell, G. \& Duncan, W. D. 1989, ApJ, 340, L69

Weintraub, D. A., Kastner, J. H. \& Bary, J. S. 2000, ApJ, 541, 767

Wyatt, M. C., et. al. 1999, ApJ, 527, 918

Zuckerman, B., \& Becklin, E. E. 1993, ApJ, 406, L25

Zuckerman, B., Forveille, T., \& Kastner, J. H. 1995, Nature, 373, 494 